INPLASY

PROTOCOL

To cite: Dang et al. Efficacy and safety of warm acupuncture in the treatment of ankylosing spondylitis: A protocol for systematic review and meta-analysis. Inplasy protocol 2020110096. doi: 10.37766/inplasy2020.11.0096

Received: 21 November 2020

Published: 22 November 2020

Corresponding author: Sha Dang

1950859817@qq.com

Author Affiliation:

Shaanxi University of

Traditional Chinese Medicine, Xianyang, Shaanxi 712000, China

Support: National Foundation of China.

Review Stage at time of this submission: Preliminary searches.

Conflicts of interest: None.

\section{Efficacy and safety of warm acupuncture in the treatment of ankylosing spondylitis: A protocol for systematic review and meta-analysis}

Dang, S1; Ren, YY2; Zhao, BY3; Meng, XW4; Zhang, CY5.

Review question / Objective: In this study, the clinical efficacy and safety of warm acupuncture in the treatment of ankylosing spondylitis were further systematically evaluated through a comprehensive literature search, To provide the latest evidence-based medical evidence for the clinical treatment of ankylosing spondylitis.

Condition being studied: Psychological problems such as anxiety and depression, but also suffer from diseases of the cardiovascular system, respiratory system, digestive system and reproductive system. Current drug treatments for ankylosing spondylitis generally include non-steroidal antiinflammatory drugs (NSAIDs), disease-modifying antirheumatic drugs (DMARDs), which can improve the symptoms of AS. However, the adverse effects of these drugs on cardiovascular, gastric, and renal function should not be ignored. To sum up, it is an urgent problem to find a safer non-therapeutic intervention with less side effects for patients with ankylosing spondylitis. Studies have shown that warm acupuncture can significantly improve the symptoms of patients with ankylosing spondylitis, play an antiinflammatory and analgesic role, as well as enhance their immunity.

INPLASY registration number: This protocol was registered with the International Platform of Registered Systematic Review and Meta-Analysis Protocols (INPLASY) on 22 November 2020 and was last updated on 22 November 2020 (registration number INPLASY2020110096).

\section{INTRODUCTION}

Review question / Objective: In this study, the clinical efficacy and safety of warm acupuncture in the treatment of ankylosing spondylitis were further systematically evaluated through a comprehensive literature search, To provide the latest evidence-based medical evidence for the clinical treatment of ankylosing spondylitis. 
Rationale: Ankylosing spondylitis can lead to anxiety, depression and other psychological problems. Studies have shown that warm acupuncture can effectively improve the symptoms of ankylosing spondylitis. As far as we know, there is no systematic review of whether warm acupuncture therapy is safe and effective in the treatment of ankylosing spondylitis. Therefore, we use this program to comprehensively evaluate the efficacy of warm acupuncture on patients with ankylosing spondylitis

Condition being studied: Psychological problems such as anxiety and depression, but also suffer from diseases of the cardiovascular system, respiratory system, digestive system and reproductive system. Current drug treatments for ankylosing spondylitis generally include non-steroidal anti-inflammatory drugs (NSAIDs), diseasemodifying antirheumatic drugs (DMARDs), which can improve the symptoms of AS. However, the adverse effects of these drugs on cardiovascular, gastric, and renal function should not be ignored. To sum up, it is an urgent problem to find a safer nontherapeutic intervention with less side effects for patients with ankylosing spondylitis. Studies have shown that warm acupuncture can significantly improve the symptoms of patients with ankylosing spondylitis, play an anti-inflammatory and analgesic role, as well as enhance their immunity.

\section{METHODS}

Search strategy: Until October 23, 2020, the Chinese biomedical literature database, Chongqing VIP, CNKI, Wanfang, Web of Science, Cochrane Library, PubMed, EMBASE and other databases were searched, and the literature on. warm acupuncture intervention for Ankylosing spondylitis, was retrieved Keywords: Ankylosing spondylitis, warm acupuncture, random English search results include 'Ankylosing spondylitis' and' warm acupuncture or warm acupuncture '.This study does not limit the scope of language retrieval.In addition, we manually searched other literature, as well as unpublished research and conference materials.If the retrieved report data is unknown or missing, we will contact the author via email.

Participant or population: The patients included in this study were all patients with ankylosing spondylitis and met the clinical diagnostic criteria for ankylosing spondylitis without age or race restrictions.

Intervention: Patients in the treatment group were mainly treated with warm acupuncture and moxibustion, while patients in the control group were included with normal acupuncture or any intervention therapy other than warm acupuncture.

Comparator: All included patients met the diagnostic criteria of ankylosing spondylitis "regardless of age or race" as amended by the American College of Rheumatology in 1984.

Study designs to be included: A randomized controlled trial (RCTS) study of warm acupuncture in the treatment of ankylosing spondylitis, published in any language.

Eligibility criteria: In addition to individual case reports, reviews, empirical summaries of animal studies and non-randomized controlled trials. This study will include all randomized controlled trials of warm acupuncture therapy in patients with ankylosis, regardless of language or publication status.

Information sources: 8 electronic databases including PubMed, Web of Science, the Cochrane Database, EMBASE, China Knowledge Network (CNKI), Wanfang Data Knowledge Service Platform, VIP Chinese Science and Technology Periodical Database (VIP) and China Biomedical Literature (CBM) Database

Main outcome(s): Activity index of Bass ankylosing spondylitis disease. 
Additional outcome(s): 1.Finger-to-floor distance (FFD); 2. occiput to wall distance (OWD); 3.ESR,CRP; 4.Adverse reaction.

Data management: Two reviewers independently screened the literature, extracted the date, and cross-checked.If there were differences, they would discuss or listen to a third party to resolve them. The extracted data mainly include: basic characteristics: author, year of publication, sample size, course of treatment, outcome indicators, follow-up, etc. methodological characteristics: random allocation method, random scheme concealment, blind method, etc.

Quality assessment / Risk of bias analysis: The two reviewers conducted a rigorous methodological quality assessment of the methodological characteristics of the included studies by referring to the Cochrane Collaborative Bias Risk Assessment tool.

Strategy of data synthesis: RevMan5.3 software was used for data analysis. Relative risk (RR) was used as the effect analysis statistic for binary variables, mean difference (MD) was used as the effect analysis system for continuous variables, and $95 \%$ confidence interval $(95 \% \mathrm{Cl})$ was used for interval estimation. $\mathrm{Na}$ into the heterogeneity between the results using chi-square test analysis (alpha test level = 0.1 ), and combining with quantitative judgment $\mathbf{I} 2$ heterogeneity is big is small, if $12<50 \%$, show good homogeneity between the various research, using the fixed effects model, if $1250 \%$ or higher, argues that the statistical heterogeneity between the results of the study is larger, should further analyze sources of heterogeneity, and the random effects model. If significant clinical heterogeneity exists, sensitivity analysis is used, or descriptive analysis only.

Subgroup analysis: Subgroup analysis will be handled according to the differences in massage methods, patient conditions, and control.
Sensibility analysis: Sensitivity analyses will be performed to verify the robustness of the review conclusions. The impacts of study design, methodological quality, and missing data will be evaluated. Sensitivity analyses were planned by studies considered being at low risk of bias.

Language: No restriction.

Country(ies) involved: China.

Keywords: warm acupuncture, Ankylosing spondylitis, protocol, systematic review.

Contributions of each author:

Author 1 - Sha Dang - The author drafted the manuscript.

Email: 1950859817@qq.com

Author 2 - YuanYuan Ren - The author gave guidance.

Email: 1454331009@qq.com

Author 3 - BoYi Zhao.

Email: 947872338@qq.com

Author 4 - XiangWei Meng.

Email: 906461053@qq.com

Author 5 - ChaoYang Zhang.

Email: 921271398@qq.com 